\title{
Basic science (April 2005)
}

Articles in this list have been selected from material published since April 2005. They represent recommended reading only.

When available the citations are linked to the abstract held on PubMed (Medline) and CrossRef.

1. Adebamowo CA, Cho E, Sampson L, Katan MB, Spiegelman D, Willett WC, Holmes MD. Dietary flavonols and flavonol-rich foods intake and the risk of breast cancer [Abstract]. Int $J$ Cancer 2005; 114: 628-633.

2. Allen NE, Roddam AW, Allen DS, Fentiman IS, Silva ID, Peto J, Holly JMP, Key TJ. A prospective study of serum insulin-like growth factor-I (IGF-I), IGF-II, IGF-binding protein-3 and breast cancer risk [Abstract]. Br J Cancer 2005; 92: 1283-1287.

3. Arturi F, Ferretti E, Presta I, Mattei T, Scipioni A, Scarpelli D, Bruno R, Lacroix L, Tosi E, Gulino A, Russo D, Filetti S. Regulation of iodide uptake and sodium/iodide symporter expression in the MCF-7 human breast cancer cell line [Abstract]. J Clin Endocrinol Metab 2005; 90: 2321-2326.

4. Astolfi A, Landuzzi L, Nicoletti G, De Giovanni C, Croci S, Palladini A, Ferrini S, lezzi M, Musiani P, Cavallo F, Forni G, Nanni P, Lollini PL. Gene expression analysis of immune-mediated arrest of tumorigenesis in a transgenic mouse model of HER-2/neu-positive basal-like mammary carcinoma [Abstract]. Am J Pathol 2005; 166: 1205-1216.

5. Barnes CJ, Li F, Talukder AH, Kumar R. Growth factor regulation of a $26 \mathrm{~S}$ proteasomal subunit in breast cancer [Abstract]. Clin Cancer Res 2005; 11: 2868-2874.

6. Barre B, Vigneron A, Coqueret O. The STAT3 transcription factor is a target for the Myc and riboblastoma proteins on the Cdc25A promoter [Abstract]. J Biol Chem 2005; 280: 15673-15681.

7. Bean GR, Scott V, Yee L, Ratliff-Daniel B, Troch MM, Seo P, Bowie ML, Marcom PK,

Publication date $30 / 06 / 05$

BCO/480/2005/JW
Slade J, Kimler BF, Fabian CJ, Zalles CM, Broadwater G, Baker JC, Wilke LG, Seewaldt VL. Retinoic acid receptor-beta 2 promoter methylation in random periareolar fine needle aspiration [Abstract]. Cancer Epidemiol Biomark Prev 2005; 14: 790-798.

8. Beckers J, Herrmann F, Rieger S, Drobyshev AL, Horsch $M$, de Angelis MH, Seliger B. Identification and validation of novel ERBB2 (HER2, NEU) targets including genes involved in angiogenesis [Abstract]. Int $J$ Cancer 2005; 114: 590-597.

9. Berquin IM, Pang B, Dziubinski ML, Scott LM, Chen YQ, Nolan GP, Ethier SP. Y-box-binding protein 1 confers EGF independence to human mammary epithelial cells [Abstract]. Oncogene 2005; 24: 3177-3186.

10. Bjornstrom L, Sjoberg M. Mechanisms of estrogen receptor signaling: convergence of genomic and nongenomic actions on target genes [Abstract]. Mol Endocrinol 2005; 19: 833-842.

11. Bosetti C, Spertini L, Parpinel M, Gnagnarella P, Lagiou P, Negri E, Franceschi S, Montella M, Peterson J, Dwyer J, Giacosa A, La Vecchia C. Flavonoids and breast cancer risk in Italy [Abstract]. Cancer Epidemiol Biomark Prev 2005; 14: 805-808.

12. Chavez-MacGregor M, Elias SG, OnlandMoret NC, van der Schouw YT, Van Gils CH, Monninkhof E, Grobbee DE, Peeters PHM. Postmenopausal breast cancer risk and cumulative number of menstrual cycles [Abstract]. Cancer Epidemiol Biomark Prev 2005; 14: 799-804.

13. Colditz GA. Epidemiology and prevention of breast cancer [Abstract]. Cancer Epidemiol Biomark Prev 2005; 14: 768-772.

14. Diermeier S, Horvath G, Knuechel-Clarke R, Hofstaedter F, Szollosi J, Brockhoff G. Epidermal growth factor receptor coexpression modulates susceptibility to Herceptin in HER2/neu overexpressing breast cancer cells via specific 
erbB-receptor interaction and activation [Abstract]. Exp Cell Res 2005; 304: 604-619.

15. Dowsett M, Ebbs SR, Dixon JM, Skene A, Griffith C, Boeddinghaus I, Salter J, Detre S, Hills M, Ashley S, Francis S, Walsh G, Smith IE. Biomarker changes during neoadjuvant anastrozole, tamoxifen, or the combination: influence of hormonal status and HER-2 in breast cancer - a study from the IMPACT trialists [Abstract]. J Clin Oncol 2005; 23: 2477-2492.

16. Gomez-Garcia EB, Ambergen T, Blok MJ, van den Wijngaard $A$. Patients with an unclassified genetic variant in the BRCA1 or BRCA2 genes show different clinical features from those with a mutation [Abstract]. J Clin Oncol 2005; 23: 2185-2190.

17. Gordon AH, O'Keefe RJ, Schwarz EM, Rosier RN, Puzas JE. Nuclear factor-kappa B-dependent mechanisms in breast cancer cells regulate tumor burden and osteolysis in bone [Abstract]. Cancer Res 2005; 65: 3209-3217.

18. Guo SC, Hakimi MA, Baillat D, Chen XW, Farber MJ, Klein-Szanto AJP, Cooch NS, Godwin AK, Shiekhattar R. Linking transcriptional elongation and messenger RNA export to metastatic breast cancers [Abstract]. Cancer Res 2005; 65: 3011-3016.

19. Gutierrez MC, Detre S, Johnston S, Mohsin SK, Shou JN, Allred DC, Schiff R, Osborne CK, Dowsett M. Molecular changes in tamoxifenresistant breast cancer: relationship between estrogen receptor, HER-2, and p38 mitogenactivated protein kinase [Abstract]. $\mathrm{J}$ Clin Oncol 2005; 23: 2469-2476.

20. Huang JW, Shiau CW, Yang YT, Kulp SK, Chen KF, Brueggemeier RW, Shapiro CL, Chen CS. Peroxisome proliferator-activated receptor gamma-independent ablation of cyclin D1 by thiazolidinediones and their derivatives in breast cancer cells [Abstract]. Mol Pharmacol 2005; 67: 1342-1348.

21. Irie A, Yamauchi A, Kontani K, Kihara M, Liu D, Shirato Y, Seki M, Nishi N, Nakamura T, Yokomise H, Hirashima M. Galectin-9 as a prognostic factor with antimetastatic potential in breast cancer [Abstract]. Clin Cancer Res 2005; 11: 2962-2968.

22. Itoh T, Karlsberg K, Kijima I, Yuan YC, Smith D, Ye JJ, Chen S. Letrozole-, anastrozole-, and tamoxifen-responsive genes in MCF-7aro cells: a microarray approach [Abstract]. Mol Cancer Res 2005; 3: 203-218.

23. Johansson EM, Kannius-Janson M, Gritli-Linde A, Bjursell G, Nilsson J. Nuclear factor 1-C2 is regulated by prolactin and shows a distinct expression pattern in the mouse mammary epithelial cells during development [Abstract]. Mol Endocrinol 2005; 19: 992-1003.

24. Kaklamani VG, Baddi L, Liu JJ, Rosman D, Phuka'n S, Bradley C, Hegarty C, McDaniel B, Rademaker A, Oddoux C, Ostrer H, Michel LS, Huang H, Chen Y, Ahsan H, Offit K, Pasche B. Combined genetic assessment of transforming growth factor-beta signaling pathway variants may predict breast cancer risk [Abstract]. Cancer Res 2005; 65: 3454-3461.

25. Kim K, Barhoumi R, Burghardt R, Safe S. Analysis of estrogen receptor alpha-Sp1 interactions in breast cancer cells by fluorescence resonance energy transfer [Abstract]. Mol Endocrinol 2005; 19: 843-854.

26. Kong G, Kim HT, Wu K, DeNardo D, Hilsenbeck SG, Xu XC, Lamph WW, Bissonnette $R$, Dannenberg AJ, Brown PH. The retinoid $X$ receptor-selective retinoid, LGD1069, downregulates cyclooxygenase-2 expression in human breast cells through transcription factor crosstalk: implications for molecular-based chemoprevention [Abstract]. Cancer Res 2005; 65: 3462-3469.

27. Lee BC, Lee TH, Zagozdzon R, Avraham S, Usheva A, Avraham HK. Carboxyl-terminal Src kinase homologous kinase negatively regulates the chemokine receptor CXCR4 through YY1 and impairs CXCR4/CXCL12 (SDF-1 alpha)-mediated breast cancer cell migration [Abstract]. Cancer Res 2005; 65: 2840-2845.

28. Lee KM, Choi JY, Park SK, Chung HW, Ahn B, Yoo KY, Han W, Noh DY, Ahn SH, Kim H, Wei QY, Kang DH. Genetic polymorphisms of ataxia telangiectasia mutated and breast cancer risk [Abstract]. Cancer Epidemiol Biomark Prev 2005; 14: 821-825.

29. Lee KW, Ma LQ, Yan XM, Liu BR, Zhang XK, Cohen P. Rapid apoptosis induction by IGFBP-3 involves an insulin-like growth factor-independent nucleomitochondrial translocation of RXR alpha/Nur77 [Abstract]. J Biol Chem 2005; 280: 16942-16948.

30. Lee SA, Ndisang D, Patel C, Dennis JH, Faulkes DJ, D'Arrigo C, Samady L, FarooquiKabir S, Heads RJ, Latchman DS, BudhramMahadeo VS. Expression of the Brn-3b transcription factor correlates with expression of HSP-27 in breast cancer biopsies and is required for maximal activation of the HSP-27 promoter [Abstract]. Cancer Res 2005; 65: 3072-3080.

31. Lee SO, Nadiminty N, Wu XX, Lou W, Dong Y, Ip C, Onate SA, Gao AC. Selenium disrupts estrogen signaling by altering estrogen receptor expression and ligand binding in human breast cancer cells [Abstract]. Cancer Res 2005; 65: 3487-3492. 
32. Ling XY, Arlinghaus RB. Knockdown of STAT3 expression by RNA interference inhibits the induction of breast tumors in immunocompetent mice [Abstract]. Cancer Res 2005; 65: 2532-2536.

33. Medina D, Kittrell FS, Hill J, Shepard A, Thordarson G, Brown P. Tamoxifen inhibition of estrogen receptor-alpha-negative mouse mammary tumorigenesis [Abstract]. Cancer Res 2005; 65: 3493-3496.

34. Moggs JG, Murphy TC, Lim FL, Moore DJ, Stuckey R, Antrobus K, Kimber I, Orphanides G. Anti-proliferative effect of estrogen in breast cancer cells that re-express ER alpha is mediated by aberrant regulation of cell cycle genes [Abstract]. J Mol Endocrinol 2005; 34: 535-551.

35. Murphy LC, Peng B, Lewis A, Davie JR, Leygue E, Kemp A, Ung K, Vendetti M, Shiu R. Inducible upregulation of oestrogen receptor-beta 1 affects oestrogen and tamoxifen responsiveness in MCF7 human breast cancer cells [Abstract]. J Mol Endocrinol 2005; 34: 553-566.

36. Nabha SM, Glaros S, Hong M, Lykkesfeldt AE, Schiff R, Osborne K, Reddy KB. Upregulation of PKC-delta contributes to antiestrogen resistance in mammary tumor cells [Abstract]. Oncogene 2005; 24: 3166-3176.

37. Narayanan R, Edwards DP, Weigel NL. Human progesterone receptor displays cell cycledependent changes in transcriptional activity [Abstract]. Mol Cell Biol 2005; 25: 2885-2898.

38. Park KJ, Krishnan V, O'Malley BW, Yamamoto Y, Gaynor RB. Formation of an IKK alpha-dependent transcription complex is required for estrogen receptor-mediated gene activation [Abstract]. Mol Cell 2005; 18: 71-82.

39. Silvera SAN, Jain $M$, Howe GR, Miller $A B$, Rohan TE. Dietary carbohydrates and breast cancer risk: a prospective study of the roles of overall glycemic index and glycemic load [Abstract]. Int J Cancer 2005; 114: 653-658.

40. Timpson P, Lynch DK, Schramek D, Walker F, Daly RJ [Abstract]. Cortactin overexpression inhibits ligand-induced down-regulation of the epidermal growth factor receptor. Cancer Res 2005; 65: 3273-3280.
41. Tripathi MK, Misra S, Khedkar SV, Hamilton N, Irvin-Wilson C, Sharan C, Sealy L, Chaudhuri G. Regulation of BRCA2 gene expression by the SLUG repressor protein in human breast cells [Abstract]. J Biol Chem 2005; 280: 17163-17171.

42. Tworoger SS, Missmer SA, Barbieri RL, Willett WC, Colditz GA, Hankinson SE. Plasma sex hormone concentrations and subsequent risk of breast cancer among women using postmenopausal hormones [Abstract]. J Natl Cancer Inst 2005; 97: 595-602.

43. Valabrega G, Montemurro F, Sarotto I, Petrelli A, Rubini P, Tacchetti C, Aglietta M, Comoglio PM, Giordano S. TGF alpha expression impairs trastuzumab-induced HER2 downregulation [Abstract]. Oncogene 2005; 24: 3002-3010.

44. Wang W, Huper G, Guo YQ, Murphy SK, Olson JA, Marks JR. Analysis of methylation-sensitive transcriptome identifies GADD45a as a frequently methylated gene in breast cancer [Abstract]. Oncogene 2005; 24: 2705-2714.

45. Webster LR, Bilous AM, Willis L, Byth K, Burgemeister FC, Salisbury ELC, Clarke CL, Balleine RL. Histopathologic indicators of breast cancer biology: insights from population mammographic screening. $\mathrm{Br} J$ Cancer 2005; 92: 1366-1371.

46. Wu Q, Ding W, Mirza A, Van Arsdale T, Wei I, Bishop WR, Basso A, McClanahan T, Luo L, Kirschmeier P, Gustafson E, Hernandez M, Liu SX. Integrative Genomics revealed RAI3 is a cell growth-promoting gene and a novel p53 transcriptional target [Abstract]. J Biol Chem 2005; 280: 12935-12943.

47. Zheng ZY, Bay BH, Aw SE, Lin VCL. A novel antiestrogenic mechanism in progesterone receptortransfected breast cancer cells [Abstract]. $\mathrm{J}$ Biol Chem 2005; 280: 17480-17487.

\author{
Prepared by \\ R. Sutherland, J. Scorer \\ Cancer Research Program \\ Garvan Institute of Medical Research \\ Darlinghurst, NSW, Australia
}

